\title{
A Computer Modeling Study of the Localization of Cardiac Optical Mapping at Discrete Depths Below the Tissue Surface
}

\author{
VK Ramshesh, JH Dumas, SB Knisley \\ University of North Carolina at Chapel Hill, Chapel Hill, USA
}

\begin{abstract}
Depth and radius of the region interrogated by cardiac optical mapping with a laser beam depend on photon travel inside the heart. It would be useful to limit range of depth and radius interrogated. Here the effects of a condensing lens to concentrate laser light at a target depth in the heart was modeled. Monte Carlo computer simulations that incorporated a $0.55 \mathrm{NA}$ lens in air and absorption and scattering of $488 \mathrm{~nm}$ laser light in 3-d cardiac tissue indicated the distribution of excitation light fluence. A subsequent computer simulation incorporating absorption and scattering of transmembrane voltage-sensitive fluorescence (669 $\mathrm{nm})$ indicated locations in tissue from which fluorescence photons exiting the tissue surface originated. The results indicate the heart can be interrogated at a discrete depth below the surface of myocardium with a condensing lens, although resolution is limited. This may be applicable to laser scanner systems used for cardiac optical mapping.
\end{abstract}

\section{Introduction}

In cardiac optical mapping with transmembrane voltage-sensitive or green emitting calcium-sensitive fluorescent dye, excitation light (typically blue or green wavelengths) is directed onto the heart. Fluorescence light is collected after the fluorescence photons exit the heart surface in an epi-illumination mode. One of the limitations of conventional optical mapping is that it records events at multiple sites distributed near the tissue surface but cannot record at discrete depths inside the tissue. Another limitation of optical mapping is the inability to interrogate a microscopic region of tissue. The interrogated region is in the order of $1 \mathrm{~mm}$ due to the scattering of excitation and emission photons inside the tissue. [1] It may be possible to achieve three dimensional optical mapping and microscopic interrogation by use of laser excitation light and a high numerical aperture lens. The lens may concentrate excitation light in a small region of the tissue at a given depth allowing microscopic interrogation at a discrete depth.

\section{Methods}

\subsection{Launch of photons with a lens}

The use of a convex lens to concentrate the excitation light was simulated. Light beams representing a radial section of a conical illumination volume were launched onto the cardiac tissue (Figure 1). The effects of the section expressed in a radial coordinate system represented the effects of a full conical illumination volume. Beam orientations and positions were calculated from the lens diameter, focal length and distance between the lens and the tissue surface. Beam intensities were adjusted for uniform illumination. A lens having a diameter $2.5 \mathrm{~cm}$ and focal length $1.9 \mathrm{~cm}$, which represented a lens with a numerical aperture of 0.55 in air, was chosen. The lens could be positioned at different distances from the surface of the tissue to produce alterations in target depth. For the excitation light, simulations were performed with optical properties of tissue for light having wavelength of $488 \mathrm{~nm}$. To study the fluorescence propagation, simulations were performed with optical properties of tissue for light having a wavelength of $669 \mathrm{~nm}$. The $669 \mathrm{~nm}$ wavelength was selected to represent the emitted fluorescence because this is near the wavelength of peak fluorescence emitted from the transmembrane voltage-sensitive dye, di-4-ANEPPS.

\subsection{Excitation light propagation}

We used a Monte Carlo model to simulate the propagation within the tissue of excitation light from each beam and to determine the excitation light fluence inside the tissue. [2] The tissue was assumed homogenous and cylindrically symmetric. The simulation recorded values proportional to fluence in each grid element as the summed weights of photons absorbed per unit area of the element normalized to total weights of launched photons. 
The recording of fluence was performed with a homogenous grid system set up in depth and radial coordinates. When a sufficient number of photons $(270,000)$ were launched and their absorption's were recorded, the resulting fluence distribution in the tissue approached a stable distribution.

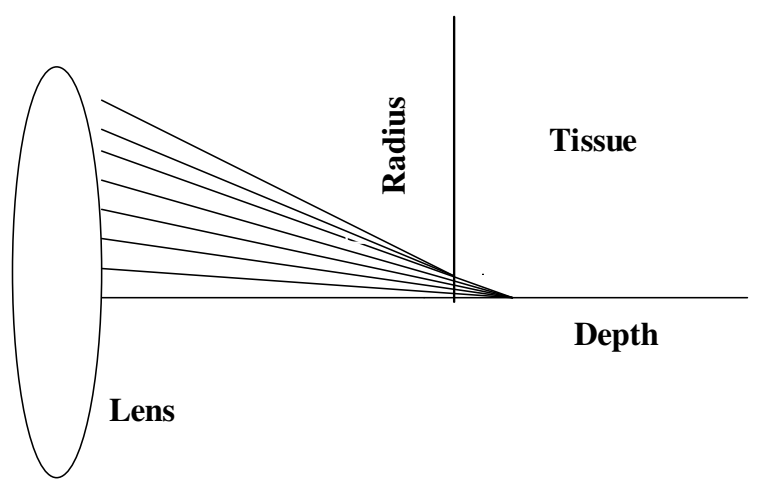

Figure 1. Diagram of radial section of illumination

\subsection{Fluorescence light propagation}

Each grid element was regarded as a fluorescence point source. The weight of a fluorescence photon launched from each grid element was proportional to the fluence of the excitation light for that grid element. During this simulation, weights of all fluorescence photons that exited the top surface of the tissue and the locations of their origin in the tissue were recorded. From these, we determined the total of weights of all fluorescence photons that exited, which can be considered proportional to the photons collected with a large area collector such as a photomultiplier tube. Using the recorded locations, we determined specific regions inside the tissue from which a given percentage of the total fluorescence originated.

\section{Results}

\subsection{Fluence of excitation light in tissue}

Figure 2 shows the distribution of fluence of $488 \mathrm{~nm}$ laser excitation light. The distance between the lens and tissue was adjusted to produce a target depth of $300 \mu \mathrm{m}$ inside the tissue. Each contour indicates the depths and radii at which fluence inside the tissue had a constant value. The maximum fluence was close to the target depth and at an extremely small radius $(<1 \mu \mathrm{m})$. This indicated that the maximum fluence occurred very close to the optical axis.

\subsection{Fluorescence collection}

Figure 3 shows the fluorescence distribution for 488 $\mathrm{nm}$ laser excitation light. The target depth was $300 \mu \mathrm{m}$ inside the tissue. The graph shows radial and depth dimensions of regions of tissue from which a certain percentage of the total collected fluorescence photons originated and from which the collected photons had the greatest weights. Contours have been plotted for percentages 80 60, 40 and 20. Regions at various depths contributed to the collected fluorescence. The photons collected with the greatest weights originated in a small region (20\% contour) that included the target depth. However when a greater percentage of the total collected fluorescence is considered, the region from which fluorescence photons originated is larger ( $80 \%$ contour).

\section{Discussion}

An interesting finding is that the fluence graph (Figure 2) shows that the region containing the brightest fluence occurred below the tissue surface and had a limited size. Also, the collected fluorescence with greatest individual photon weights originated in regions below the tissue surface (e.g., $20 \%$ contour in Figure 3). This indicates that a condensing lens may help in distinguishing signals from regions below the heart surface. The results may be applicable in optical mapping to interrogate microscopic regions of tissue at a discrete depth.

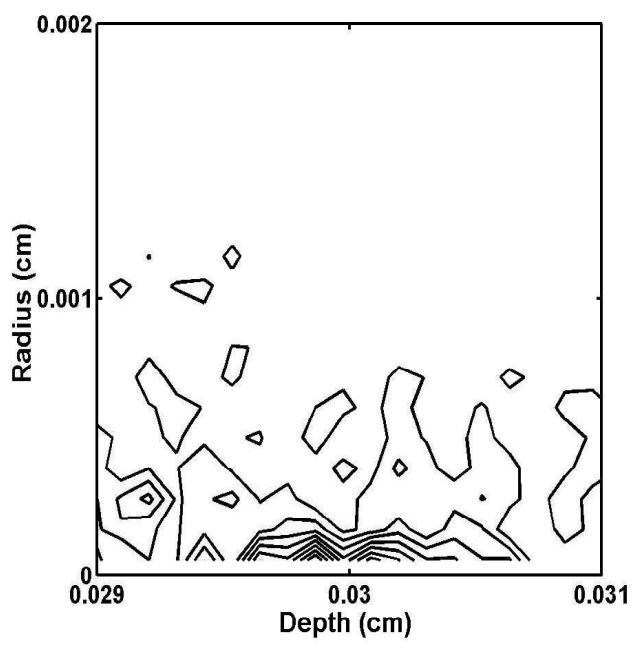

Figure 2. Fluence graph for $488 \mathrm{~nm}$ excitation.

The laser and lens were to the left of the graph, coaxial with the X-axis. Contours are shown for values 0.2 to 0.9 times the maximum fluence. The greatest fluence was near the target depth. 


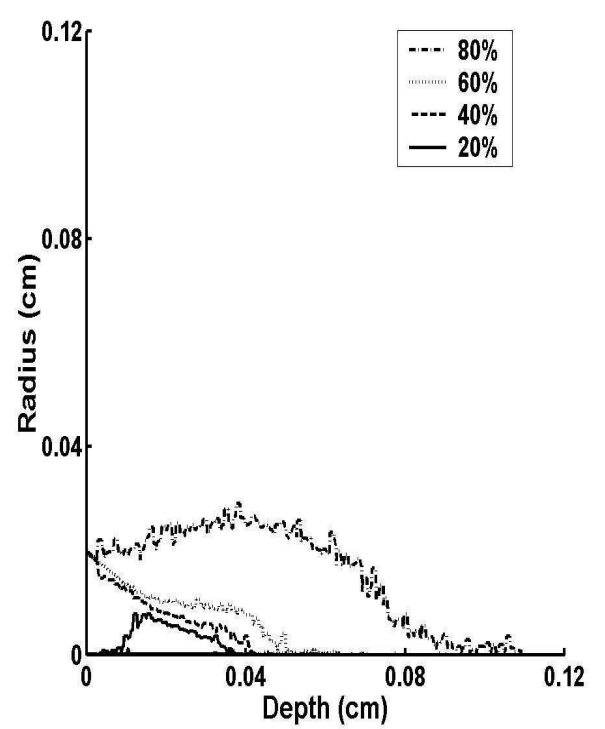

Figure 3. Regions interrogated for $488 \mathrm{~nm}$ excitation and $669 \mathrm{~nm}$ fluorescence emission. The laser and lens were to the left of the graph, coaxial with the $\mathrm{x}$-axis.

\section{Acknowledgements}

Supported by National Institutes of Health Grants HL52003, HL67728 and RR11718, and American Heart Association Grant 9740173N.

\section{References}

[1] Ding L, Splinter R, Knisley SB. Quantifying spatial localization of optical mapping with monte carlo simulations. IEEE Transactions on Biomedical Engineering 2001; 48:1098-1107.

[2] Wang L, Jacques SL, Zheng L. MCML--Monte Carlo modeling of light transport in multi-layered tissues. Comput Methods Programs Biomed 1995; 47:131-146.

Address for correspondence.

Venkat K Ramshesh

Department of Biomedical Engineering

CB\# 7575, 152 Macnider Hall

Chapel Hill, NC 27599-7575

venkat@email.unc.edu 
